ARTIGO ORIGINAL

\title{
SOROPREVALÊNCIA DE INFECÇÃO HUMANA POR Trypanosoma cruzi EM UMA ÁREA RURAL DO SUL DO BRASIL
}

\author{
Adelita Campos Araújo1, Suelen Costa Rodrigues ${ }^{1}$, Andreá de Fátima Silva \\ Rezende $^{2}$, Marcos Marreiro Villela ${ }^{1}$ e Sibele Borsuk ${ }^{1}$
}

\section{RESUMO}

A doença de Chagas é causada pelo protozoário Trypanosoma cruzi. Apesar de ser endêmica na América Latina, há poucos estudos de soroprevalência de infecção por T. cruzi em áreas rurais, onde os indivíduos têm menos acesso à informação sobre esta enfermidade. Neste trabalho, teve-se como objetivo investigar a soroepidemiologia de T. cruzi em uma população humana rural do município de Pelotas, RS. Participaram 227 usuários de uma Unidade Básica de Saúde (UBS) da localidade Cerrito Alegre-RS ( $3^{\circ}$ distrito de Pelotas-RS). Amostras de sangue foram coletadas e os soros testados quanto à presença de anticorpos anti-T. cruzi por meio de Imunoensaio Quimioluminescente de Micropartículas e, quando reagentes, confirmados via Imunofluorescência Indireta. O levantamento dos fatores de risco associados à presença da parasitose deu-se por meio de um questionário semiestruturado. Na população da região rural avaliada foi encontrado o índice de $2,7 \%$ de soropositividade para $T$. cruzi. Dentre os fatores de risco avaliados, dois apresentaram diferença estatística significativa: o tipo de moradia ( $\mathrm{P}$ 0,0093 ), com maior risco morar ou ter morado em casa de pau-a-pique, barro e madeira (OR 46,9), e o fato de já ter sido picado pelo vetor (P 0,0309 e OR 14,5).

DESCRITORES: Doença de Chagas; fatores de risco; soroepidemiologia.

\section{ABSTRACT}

Seroprevalence of human infection with Trypanosoma cruzi in a rural area of southern Brazil

Chagas disease is caused by the protozoan Trypanosoma cruzi. Although Latin America is defined as endemic, there are few studies in relation to seroprevalence of T. cruzi infection in rural areas, where the individuals do not have information on this disease. The objective was to investigate the seroepidemiology of T. cruzi in a rural human population of the city of Pelotas,

1. Programa de Pós-Graduação em Parasitologia, Instituto de Biologia, Universidade Federal de Pelotas (UFPel), Capão do Leão, RS, Brasil.

2. Programa de Pós-Graduação em Biotecnologia, Centro de Desenvolvimento Tecnológico, UFPel, Capão do Leão, RS, Brasil.

Correspondência para: Sibele Borsuk, Laboratório de Pesquisa em Doenças Infecciosas, Centro de Desenvolvimento Tecnológico, Universidade Federal de Pelotas (UFPel) - Campus Universitário s/n, Prédio 19, Pelotas, RS, Brasil, CEP 96010-900. E-mail: sibeleborsuk@gmail.com 
Brazil. A total of 227 individual users of a Basic Health Unit (BHU) of Cerrito Alegre, (third district of Pelotas, RS) were included in this study. Blood samples were collected and sera were tested for antibodies against $T$. cruzi through Chemiluminescent Microparticle Immunoassay assay and confirmation by Indirect Immunofluorescence test. The risk factors associated with the presence of the parasite were evaluated through a semi-structured questionnaire. The population evaluated had $2.7 \%$ seropositivity for $T$. cruzi. Among the risk factors, two were statistically different: the kind of housing ( $\mathrm{p}=0.0093)$, with the greatest risk to live or have lived in a house of wattle-and-daub, clay, wood (OR 46.9) and the fact that the individual had been bitten by the vector $(\mathrm{p}=0.0309$ and $\mathrm{OR} 14.5)$.

KEY WORDS: Chagas disease; risk factors; seroepidemiology.

\section{INTRODUÇÃO}

A doença de Chagas (DC), conhecida também como Tripanossomíase Americana, é causada pelo protozoário flagelado Trypanosoma cruzi e caracteriza-se por ser uma infecção parasitária sistêmica com tendência à cronificação (Ramos et al., 2012). A referida enfermidade foi comunicada ao mundo científico em 1909 pelo pesquisador brasileiro Carlos Ribeiro Justiniano Chagas (Chagas, 1910).

A Organização Mundial da Saúde estima que 8 milhões de pessoas estejam infectadas na América Latina por T. cruzi, região em que a DC é endêmica (Rassi et al, 2010).

A DC é predominantemente rural, mas seu padrão epidemiológico sofreu alterações em virtude da migração da população do interior para centros urbanos na busca por melhores condições de vida. Na maioria das vezes é transmitida aos seres humanos pelas fezes de insetos triatomíneos infectados por T. cruzi (via vetorial), conhecidos popularmente como insetos "barbeiros" ou "chupões", entre outros nomes, dependendo da área geográfica (OMS, 2002). Em um estudo realizado, observou-se que, desde 2007, a infestação por triatomíneos no interior dos domicílios vem aumentando em localidades rurais de algumas regiões do Brasil (Vasconcelos et al., 2013), o que caracteriza a vinda do vetor para dentro das habitações por causa do desmatamento. Cabe lembrar que a enfermidade também pode ser transmitida por via vertical (de mãe para o filho pela placenta), transfusão de sangue e ingestão de alimentos contaminados com tripomastigotas, entre outras formas (Gurgel-Gonçalves et al., 2012; Vasconcelos et al., 2013).

Um inquérito de soroprevalência de DC realizado com 104.954 crianças até 5 anos de idade de toda a área rural brasileira, exceto o estado do Rio de Janeiro, evidenciou $104(0,1 \%)$ positivas, das quais somente $32(0,03 \%)$ foram ratificadas como infectadas. Do total de crianças avaliadas, havia 20 $(0,02 \%)$ com positividade materna concomitante (sugerindo transmissão congênita), $11(0,01 \%)$ com positividade exclusivamente na criança (indício 
de provável transmissão vetorial) e uma criança positiva cuja mãe havia ido a óbito. Salienta-se que as amostras foram submetidas a testes de screening pelas técnicas de imunofluorescência indireta (IFI) e ELISA (Luquetti-Ostermayer et al., 2011).

Num estudo realizado por Moreno et al. (2010), foram analisadas amostras de sangue de 5.240 indivíduos entre 6 meses e 40 anos de idade, moradores de 192 comunidades rurais no sul de Gran Chaco na Argentina. O objetivo foi detectar, via ELISA, infecção por T. cruzi, confirmado-se com imunofluorescência indireta e hemaglutinação indireta. A soroprevalência geral foi de $5,4 \%, 7,9 \%$ e $7,5 \%$, respectivamente no norte, noroeste e oeste das áreas pesquisadas. Verificou-se que a soroprevalência para T. cruzi aumentou conforme a idade da população, especialmente na faixa acima dos 15 anos.

A DC é uma doença ainda presente no Brasil e Pelotas-RS é um local onde a enfermidade é endêmica, incluindo a eventual presença de vetores nos domicílios rurais (Priotto et al., 2014). Diante disso, percebeu-se a importância de pesquisar a população rural não apenas para conhecer os casos positivos e negativos, mas também para saber sobre seus hábitos e costumes e como esses podem contribuir para a enfermidade ou preveni-la. Assim, o presente estudo teve como objetivo investigar a soroepidemiologia de $T$. cruzi em uma população humana rural do município de Pelotas-RS.

\section{METODOLOGIA}

\section{Amostras e delineamento experimental}

Do estudo de caráter quantitativo fizeram parte usuários de uma Unidade Básica de Saúde (UBS) da localidade Cerrito Alegre-RS $\left(3^{\circ}\right.$ distrito

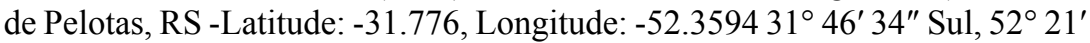
$34^{\prime \prime}$ Oeste). O levantamento dos possíveis fatores de risco associados à presença da parasitose deu-se por meio da aplicação de um questionário epidemiológico semiestruturado. Amostras de sangue foram coletadas de 227 indivíduos com idade entre 18-78 anos. Os critérios para inclusão dos sujeitos do estudo foram: ser morador do $3^{\circ}$ Distrito de Pelotas, concordar em participar da pesquisa assinando o Termo de Consentimento Livre e Esclarecido e ser maior de 18 anos. O referido projeto foi submetido à apreciação e obteve parecer favorável do Comitê de Ética da Faculdade de Medicina da Universidade Federal de Pelotas (OF. $n^{0} 36 / 12$ ). Todos os pacientes receberam o laudo do exame e, quando necessário, foi realizado atendimento de saúde por profissionais da própria UBS. O soro foi testado para a presença de anticorpos anti-T.cruzi por meio de Imunoensaio Quimioluminescente de Micropartículas (CMIA) para triagem e, quando reagente, a confirmação se deu por meio da Reação 
de Imunofluorescência Indireta (RIFI). Para o CMIA foi utilizado o ensaio ARCHITECT Chagas ${ }^{\circledR}$ (Abbott) (com protocolos designados Chemiflex). Já a RIFI foi realizada com o kit comercial Imuno-CON CHAGAS ${ }^{\circledR}$ (WAMA Diagnóstica) com critério de reatividade $1 / 30$, conforme é sugerido pelo protocolo do conjunto diagnóstico.

\section{Análise estatística}

Todos os dados obtidos por meio dos questionários foram computados no programa Epi Info Versão 6.04 (CDC). O teste de $\chi^{2}$ com intervalo de confiança de $95 \%$ foi utilizado para avaliar a soroprevalência da DC em relação aos diferentes fatores epidemiológicos conforme a Tabela.

\section{RESULTADOS}

Os dados foram coletados entre março de 2011 e novembro de 2014 e totalizaram amostras sanguíneas de 227 indivíduos. Destes, 2,7\% (6) apresentaram sororreatividade para T. cruzi (reagentes no CMIA e confirmados pela RIFI), sendo quatro do sexo feminino e dois do masculino. Os soropositivos tinham idade superior a 31 anos (Tabela 1). Acerca da moradia, cinco haviam morado em outra casa (pau-a-pique, barro ou madeira, OR 46,8987). Ainda, apenas um respondeu que havia barbeiros em sua moradia anterior, dois confirmaram ter visto o vetor em sua residência atual e um afirmou já ter sido picado pelo inseto (OR 14, 5333). Seis não possuíam escolaridade ou haviam concluído apenas o ensino fundamental. Sobre a renda familiar, um dispunha de apenas um salário mínimo e cinco de dois salários mínimos ou mais. No peridomicílio, seis relataram a presença de areia, terra e/ou fezes, dois já haviam visualizado barbeiros, seis relataram limpar suas casas diária ou semanalmente; três, limpar o peridomicílio diária ou semanalmente e três, quinzenalmente ou mais.

\section{DISCUSSÃO}

A positividade evidenciada foi semelhante à observada em outros locais no Brasil, como Rio Grande do Norte, Manaus e São Paulo, respectivamente 3,3\%, 1,9\% e 3\% (Brito et al., 2012; Carvalho et al, 2011; Magalhães et al., 2011). Foi inferior à verificada em algumas áreas do país, como: Jaguaruana, CE; Miguel Pereira, CE; Turumã-Mirim, Coari e Tefé-AM, respectivamente $0,6 \%, 1,2 \%$ e 0,8\% (Coutinho et al., 2014; Lima et al., 2015; Magalhães et al., 2011). Mostrouse superior às encontradas em Campos de Itapetininga e Encosta Ocidental da Mantiqueira Paulista-SP, em 16 municípios do RN, em seis microrregiões ao 
redor do Rio Negro-AM e em Bambuí- MG, respectivamente 10,2\%, 6,5\%, 4,5, 7,7\% (Brito et al., 2012; Carvalho et al, 2011; Coura, et al., 2013; Matos et al., 2014). Essa diversidade nos índices de soropositividade possivelmente é devida à idade dos pacientes, à origem da população estudada, à eficácia no controle do vetor e às disparidades nas técnicas diagnósticas empregadas (ELISA, IFI, Western blot) (Pedroso et al., 2012).

Tabela. Características sociodemográficas da população estudada para a presença de Trypanosoma cruzi em uma área rural da região sul do Rio Grande do Sul.

\begin{tabular}{|c|c|c|c|c|}
\hline \multirow[t]{2}{*}{ Variáveis } & \multicolumn{2}{|c|}{$\begin{array}{l}\text { Detecção de anticorpos } \\
\text { contra } T . c r u z i\end{array}$} & \multirow[t]{2}{*}{ OR } & \multirow[t]{2}{*}{$\mathrm{P}$} \\
\hline & Negativo & Positivo & & \\
\hline \multicolumn{5}{|l|}{ Idade } \\
\hline 18-31 anos & 25 & 0 & & 0.7243 \\
\hline Mais de 31 anos & 202 & 6 & & \\
\hline \multicolumn{5}{|l|}{ Sexo } \\
\hline Feminino & 148 & 4 & & 0,939 \\
\hline Masculino & 79 & 2 & & \\
\hline \multicolumn{5}{|l|}{ Escolaridade } \\
\hline Nenhuma ou $1^{\circ}$ grau completo & 225 & 6 & & 0.2259 \\
\hline $2^{\circ}$ grau completo e superior & 2 & 0 & & \\
\hline \multicolumn{5}{|l|}{ Renda } \\
\hline 01 salário mínimo & 59 & 1 & & 0.6216 \\
\hline 02 salários mínimos ou mais & 168 & 5 & & \\
\hline \multicolumn{5}{|l|}{ Tipo de moradia (mora ou já morou)? } \\
\hline Pau-a-pique/barro/madeira & 45 & 6 & 46.8987 & 0.0093 \\
\hline Alvenaria/mista & 142 & 0 & & \\
\hline \multicolumn{5}{|l|}{ Peridomicílio } \\
\hline Lixo/entulho/mato & 50 & 0 & & 0.3632 \\
\hline Areia/terra/fezes & 177 & 6 & & \\
\hline \multicolumn{5}{|l|}{ Já viu barbeiros? } \\
\hline Sim & 23 & 2 & & 0.0815 \\
\hline Não & 204 & 4 & & \\
\hline \multicolumn{5}{|l|}{ Frequência de limpeza da casa } \\
\hline Diária/semanal & 218 & 6 & & 0.7183 \\
\hline Quinzenal ou mais & 9 & 0 & & \\
\hline \multicolumn{5}{|l|}{$\begin{array}{l}\text { Frequência de limpeza do } \\
\text { peridomicílio }\end{array}$} \\
\hline Diária/semanal & 135 & 3 & & 0.6340 \\
\hline Quinzenal ou mais & 92 & 3 & & \\
\hline \multicolumn{5}{|l|}{ Já foi picado por barbeiro? } \\
\hline Sim & 4 & 1 & 14.5333 & 0.0309 \\
\hline Não & 223 & 5 & & \\
\hline
\end{tabular}


Na Tabela, está demonstrado o seguinte dado acerca da moradia dos positivos (seis): cinco haviam morado em outra casa (pau-a-pique, barro ou madeira, OR 46,8987), o que evidencia a maior possibilidade de ter tido contato com o barbeiro. Em uma investigação relacionada com o tipo de moradia em três municípios no estado do Amazonas, 89\% (1.125) da população estudada morava em casas de madeira, desse total 1,3\% (19) dos indivíduos foram positivos para $\mathrm{DC}$ em diferentes tipos de procedimentos diagnósticos (Magalhães et al., 2011). Um levantamento sociodemográfico com 28 pacientes portadores da DC demonstrou que $60,7 \%$ desses indivíduos nasceram em zona rural e metade deles ainda reside no campo. No que tange ao tipo de domicílio dos indivíduos deste estudo, 14,3\% moravam em casas de taipa, 35,6\% de bloco, 28,6\% de tijolo e 21,4\% de adobe (Carvalho et al., 2013). Esses fatores reforçam a hipótese de que alguns tipos de moradia tornam-se fatores de risco para a doença em questão. Bernardo et al. (2013) estudaram as residências associando-as com a DC confirmada em 32 mulheres. Destas, $53,1 \%$ teriam nascido em casas de madeira, $28,1 \%$ moraram anteriormente em casa de madeira e $15,6 \%$ nasceram em casa de pau-a-pique, contudo a alvenaria representou $91,8 \%$ do tipo de habitação atual.

Dentre os casos positivos deste estudo, um respondeu que havia barbeiros em sua moradia anterior, dois confirmaram ter visto o vetor em sua residência atual. Somente um afirmou já ter sido picado pelo inseto, fator de risco estatisticamente relacionado à soroprevalência de T. cruzi. Em contrapartida, quatro indivíduos relataram que haviam sido picados pelo barbeiro, mas o resultado deles foi negativo para Chagas. Isso pode ter acontecido, provavelmente, por terem sido atingidos por um inseto não triatomíneo, como um hemíptero predador ou fitófago, já que tal equívoco de identificação é comum (Villela et al., 2009a). Adicionalmente, no caso de ser realmente um vetor de T. cruzi, o inseto poderia estar negativo para a presença do protozoário ou não ter realizado a defecação sobre o hospedeiro.

Em trabalho na área rural do sul do Mato Grosso do Sul, 12 pessoas $(17,6 \%)$ conheciam o triatomíneo e $2(2,9 \%)$ disseram ter tido contato com o inseto (Corrêa et al., 2011). Brito et al. (2012) realizaram uma avaliação soroepidemiológica em uma região endêmica do Rio Grande do Norte, na qual a presença de triatomíneos dentro das residências foi relatada por $68,1 \%$ dos participantes. Isso pode ser atribuído ao fato de que muitos indivíduos até possuem conhecimento sobre a possibilidade de transmissão vetorial da parasitose, mas não fazem relação com a transmissão via dejeção dos triatomíneos e sim com a sua picada. Portanto, provavelmente este indivíduo que relatou ter sido picado sabia que tinha a DC e associou a presença da enfermidade com este episódio.

Em relação à idade, nos seis casos positivos os indivíduos tinham idade acima de 31 anos. Coutinho et al. (2014) constataram, em Miguel Pereira-CE, três indivíduos positivos com idade acima de 40 anos e um na faixa etária de 
20 a 29 anos. Carvalho et al. (2011), examinando duas microrregiões do estado de São Paulo, com 31.468 indivíduos, verificaram que na microrregião de Campos de Itapetininga a idade média entre os positivos era de 31 anos, sendo a maioria do sexo feminino (51,7\%). Estes dados estão em conformidade com o estudo aqui apresentado tanto em relação à idade média quanto ao sexo. Já na microrregião da Encosta Ocidental da Mantiqueira Paulista, a idade média dos reagentes foi de 48 anos, $75 \%$ até 58 anos, sendo a maioria pertencente ao sexo feminino (52,4\%). Brito et al. (2012), no Rio Grande do Norte, apuraram que a idade média dos soropositivos da mesorregião oeste foi de 48 anos e, no município de Caicó, de 47 anos. É importante salientar a idade, visto que se trata de pessoas infectadas provavelmente na infância ou adolescência, quando moravam em casas de madeira, barro ou pau-a-pique. Logo, percebe-se que a idade dos pacientes positivos para DC encontra-se, de modo geral, acima dos 30 anos e a ausência de casos abaixo desta idade pode ser fruto do sucesso que o Programa de Controle da Doença de Chagas obteve na região.

Embora a limpeza e a higiene geral da moradia possam ser consideradas estratégias genéricas e pouco específicas no combate aos vetores da DC (Villela et al., 2009a), elas são importantes, sobretudo quando visam à redução ou à eliminação dos locais que servem de esconderijo para os triatomíneos nos quais eles podem constituir colônias, em especial no peridomicílio. No que tange à infestação de domicílios e peridomicílios, Coutinho et al. (2014) detectaram que $18,2 \%$ das 220 casas pesquisadas apresentavam infestação no peridomícílo, $3,2 \%$ possuíam infestação domiciliar e 761 triatomíneos de diversas espécies foram capturados. Estes dados evidenciam a importância de se estudar o peridomicilio e até enfatizá-lo quando se discute a via vetorial da DC, já que este é normalmente o principal ambiente de presença de vetores em ecótopos artificiais e o local onde a população está menos atenta. Importa ainda registrar que, embora a população normalmente capture mais insetos no intradomicílio, o peridomicílio, via de regra, apresenta mais infestações e com mais insetos, mas estas são detectadas apenas por agentes que trabalham no Programa de Controle da Doença de Chagas (Villela et al., 2009b), fato também confirmado na região sul do Rio Grande do Sul (Priotto et al., 2014).

Com base nos dados encontrados, conclui-se que a população estudada, ou seja da região rural de Pelotas, possui $2,7 \%$ de soropositividade para T. cruzi. Embora este índice possa ser considerado baixo quando comparado com anterior, 28,9\% (Baruffa e Alcântara-Filho, 1985), nossos encontros diagnosticaram índices superiores $(0,9 \%)$ a inquérito realizado na mesma região, nos últimos anos, com doadores de sangue (Araújo et al., 2008). Entre os fatores de risco avaliados, foram significativos o tipo de moradia (pau-a-pique, barro, madeira) e o fato de o indivíduo já ter sido picado pelo vetor, elementos que devem ser considerados em futuras avaliações.

Partindo dessas considerações, é desejável que sigam ocorrendo análises soroepidemiológicas na região, dada a presença histórica da DC em moradores do 
sul do Rio Grande do Sul. Além disso, os indivíduos que recebem o diagnóstico da doença podem se beneficiar de acompanhamento médico, evitando eventuais danos ocasionados pela enfermidade em sua forma crônica manifesta.

\section{REFERÊNCIAS}

1. Araújo AB, Vianna EES, Berne MEA. Anti-Trypanosoma cruzi antibody detection in blood donors in the Southern Brazil. Braz J Infect Dis 12: 480-482, 2008.

2. Baruffa G, Alcantara Filho A. Inquérito entomológico e sorológico da infecção pelo T.cruzi na região sul do Rio Grande do Sul, Brasil. Ann Soc Belg Med Trop 65: 171-179, 1985.

3. Bernardo F, Ivo ML, Ferrairo MM, Fabbro JD, Pontes ERJC, Junior, MAF. Epidemiologic aspects of pregnant women with Chagas disease. J Nurs UFPE on line 7: 5250-5256, 2013.

4. Brito CRN, Sampaio GHF, Câmara ACJ, Nunes DF, Azevedo PRM, Chiari E, Galvão LMC. Seroepidemiology of Trypanosoma cruzi infection in the semiarid rural zone of the State of Rio Grande do Norte, Brazil. Rev Soc Bras Med Trop 45: 346-352, 2012.

5. Carvalho ME, Silva RA, Wanderley DMV, Barata JMS. Chagas disease Control Program in the State of São Paulo, Brazil Seroepidemiological aspects of homogeneous geographic microregions. Rev Soc Bras Med Trop 44: 85-94, 2011.

6. Carvalho RB, Silva HC, Couto, MVG, Conceição FB, Junior GR, Bastos CJC. Perfil Biossocial dos Indivíduos Portadores de Doença de Chagas atendidos no Ambulatório de Infectologia do Hospital Couto Maia, Salvador, Bahia. Rev Baiana Saúde Pública 37: 133-143, 2013.

7. Chagas C. Nova entidade mórbida do homem. Mem Inst Oswaldo Cruz 3: 220-275, 1911.

8. Corrêa NAB, Velásquez LG, Marques JM, Silva AV. Levantamento Soroepidemiológico da Leishmaniose Tegumentar e Doença de Chagas em uma Comunidade Rural da Região Sul do Estado do Mato Grosso do Sul, BRASIL. Arq Ciênc Saúde UNIPAR 15: 23-28, 2011.

9. Coura JR, Marquez MHP, Guerra JAO, Zauza PL, Miguel JC, Pereira JB. A new survey of the serology of human Trypanosoma cruzi infection in the Rio Negro microregion, Brazilian Amazon: a critical analysis. Mem Inst Oswaldo Cruz 108: 909-913, 2013.

10. Coutinho CFS, Souza-Santos R, Teixeira NFD, Georg I, Gomes TF, Boia MN, Reis NB, Maia AO, Lima MM. An entomoepidemiological investigation of Chagas disease in the state of Ceará, Northeast Region of Brazil. Cad Saúde Pública 30: 785-793, 2014.

11. Gurgel-Gonçalves R, Galvão C, Peterson AT. Geographic Distribution of Chagas Disease Vectors in Brazil Based on Ecological NicheModeling. J Trop Med 2012: 1-15, 2012.

12. Lima MM, Carvalho-Costa FA, Toma HK, Borges-Pereira J, Oliveira TG, Sarquis O. Chagas disease and housing improvement in northeastern Brazil: a cross-sectional survey. Parasitol Res 114: 1687-1692, 2015.

13. Luquetti-Ostermayer AL, Passos ADC, Silveira AC, Ferreira AW, Macedo V, Prata AR. O inquérito nacional de soroprevalência de avaliação do controle da doença de Chagas no Brasil (2001-2008). Rev Soc Bras Med Trop 44:108-121, 2011.

14. Magalhães BML, Coelho LIARC, Maciel MG, Ferreira JMBB, Umezawa ES, Coura JR, Guerra JAO, Barbosa MGV. Serological survey for Chagas disease in the rural areas of Manaus, Coari, and Tefé in the Western Brazilian Amazon. Rev Soc Bras Med Trop 44: 697702, 2011.

15. Matos CS, Júnior JES, Medeiros FAC, Furtado E, Dias, JCP. Current situation and perspectives regarding human Chagas disease in midwestern of the state of Minas Gerais, Brazil. Mem Inst Oswaldo Cruz 109: 374-378, 2014. 
16. Moreno ML, Moretti E, Basso B, Céspedes MF, Catalá SS, Gorla DE. Seroprevalence of Trypanosoma cruzi infection and vector control activities in rural communities of the southern Gran Chaco (Argentina). Acta Tropica 113: 257-262, 2010.

17. Organização Mundial da Saúde. Control of Chagas disease. WHO Technical Report Series (905). Geneva, 1-106, 2002. Disponível em: <http://apps.who.int/iris/bitstream/10665/42443/1/ WHO_TRS_905.pdf $>$. Acessado em 18 novembro 2015.

18. Pedroso D. A Retrospective Study of Chagas Disease In Blood Donors from Northwest of Rio Grande do Sul State, Brazil. Pelotas [Dissertação de Mestrado]. Programa de Pós-Graduação em Parasitologia. UFPel, 2012.

19. Priotto MCM, Santos CV, Mello F, Ferraz ML, Villela MM. Aspectos da Vigilância Entomológica da Doença de Chagas no Sul, do Rio Grande do Sul, Brasil. Rev Patol Trop 43: 228-238, 2014.

20. Ramos JM, Ponce J, Gallegos I, Flores-Chavez M, Cañavate C, Gutierrez F. Trypanosoma cruzi infection in Elche (Spain): comparison of the seroprevalence in immigrants from Paraguay and Bolivia. Pathog Glob Health 106: 102-106, 2012.

21. Rassi AJ, Rassi A, Marin-Neto JA. Chagas disease. The Lancet 375:1388-1402, 2010.

22. Vasconcelos ASOB, Freitas EC, Andrade MC, Lima MM, Pereira LS, Gomes KCMS, Coelho HLL, Oliveira MF. Chagas Disease: vector status in the city of Limoeiro do Norte, CE, during the period from 2006 to 2009. Rev Inst Adolfo Lutz 72: 295-301, 2013.

23. Villela MM, Pimenta DN, Lamounier PA, Dias JCP. Avaliação de conhecimentos e práticas que adultos e crianças têm acerca da doença de Chagas e seus vetores em região endêmica de Minas Gerais, Brasil. Cad Saúde Pública 25: 1701-1710, 2009a.

24. Villela MM, Souza JMB, Melo VP, Dias JCP. Avaliação do Programa de Controle da Doença de Chagas em relação à presença de Panstrongylus megistus na região centro-oeste do Estado de Minas Gerais, Brasil. Cad Saúde Pública 25: 907-917, 2009 b. 